\title{
Recent advances in experimental animal models of lung cancer
}

\author{
Vamshikrishna Malyla ${ }^{\dagger, 1,2}$, Keshav Raj Paudel ${ }^{\dagger, 2,3}$, Shakti D Shukla4, Chantal Donovan ${ }^{2,3,4}$, \\ Ridhima Wadhwa ${ }^{1,2}$, Sophie Pickles 2,4 , Vrushali Chimankar ${ }^{2,4}$, Priyanka Sahu ${ }^{2,4}$, Helle \\ Bielefeldt-Ohmann ${ }^{5}$, Mary Bebawy ${ }^{1}$, Philip M Hansbro*,2,3,4 \& Kamal Dua**,1,2,4 \\ ${ }^{1}$ Discipline of Pharmacy, Graduate School of Health, University of Technology Sydney, Chippendale, NSW 2007, Australia \\ ${ }^{2}$ Centre for Inflammation, Centenary Institute, Sydney, NSW 2050, Australia \\ ${ }^{3}$ School of Life Sciences, University of Technology Sydney, Sydney, NSW 2007, Australia \\ ${ }^{4}$ Priority Research Centre for Healthy Lungs \& Hunter Medical Research Institute, The University of Newcastle, Newcastle, NSW \\ 2305, Australia \\ ${ }^{5}$ Australian Infectious Diseases Research Centre, School of Chemistry \& Molecular Biosciences, The University of Queensland, St. \\ Lucia, Queensland 4072, Australia \\ *Author for correspondence: Tel.: +61 2 95656248; Philip.hansbro@uts.edu.au \\ **Author for correspondence: Tel.: +61 2 95147387; Kamal.Dua@uts.edu.au \\ $\dagger$ Authors with equal contribution
}

"Chemically induced animal models of lung cancer are increasing in importance compared with xenograft and genetically modified models due to their ease of application, rapid timeframes and efficiency in reproducibility."”

First draft submitted: 26 November 2019; Accepted for publication: 16 January 2020; Published online: 16 March 2020

Keywords: 4-[Methyl(nitroso)amino]-1-(3-pyridinyl)-1-butanone • animal models $\bullet$ cigarette smoking $\bullet$ lung cancer - non-small-cell lung cancer

Lung cancer is the leading cause of cancer-related deaths worldwide [1]. In 2018, there were 2 million new cases and 1.7 million deaths attributed to lung cancer [1]. Histopathological subtypes of lung cancer include non-small-cell lung cancer (NSCLC) and small-cell lung cancer (SCLC). NSCLC accounts for approximately $85 \%$ of lung cancer cases, which can be further divided into adenocarcinoma accounting for $38.5 \%$, squamous cell carcinoma for nearly $20 \%$ and bronchoalveolar and large cell carcinomas accounting for the rest [2]. SCLC arises from neuroendocrine cells of bronchus and constitutes approximately $15 \%$ of cases [3].

Lung cancer is typically a lethal malignancy, primarily due to delay in definitive clinical diagnosis and consequently has a poor prognosis [1]. Thus, there is an urgent need for novel and efficacious diagnosis, as well as therapeutic options. The research in this area could be accelerated by utilizing short-term animal models that recapitulate the hallmark features of lung cancer that will be valuable in pinpointing novel therapeutic targets and appropriate interventions [4]. Importantly, cigarette smoking (both active and passive) is a major contributing factor for the development of lung cancer and chronic obstructive pulmonary disease [5]. It is likely that increasing air pollution, especially in low- and middle-income countries, is also likely to contribute to lung cancer development [6]. However, the precise underlying mechanisms that drive carcinogenesis, both molecular and cellular, remain poorly understood $[4,7]$. Recent advances in genetic engineering technology, including transposon-based insertional mutagenesis, RNA interference and engineered nucleases, as well as chemically-induced preclinical animal models of lung cancer have significantly contributed to furthering our understanding of carcinogenesis and associated pathophysiology. In this commentary, we will specifically focus on the recent advances in animal models of lung cancer.

\section{Genetically modified animal models of lung cancer}

Highly specific transgenic mouse models that closely resemble the genetic and pathophysiological features of human lung cancers are now available. Tumor suppressor genes (TSGs), which play a vital role in lung cancer, are often deleted or mutated to induce lung cancer development in mice. Based on the genome database and known 
mutations, Wu et al. selected 55 TSGs and screened them for their roles in tumorigenesis using the somatic gene editing technique, clustered regularly interspaced short palindromic repeats (CRISPR/Cas9) in the Kras ${ }^{\mathrm{G} 12 \mathrm{D} /+}$ (Kirsten rat sarcoma viral oncogene homolog) mouse model of lung cancer [8]. Interestingly, of the genes Utx, Ptip, Acp5, Acacb and Clu, only when the Utx gene was deleted, tumor progression increased [8]. Consequently, the study of specific TSGs may facilitate our understanding of lung tumorigenesis and investigation of novel pharmacotherapies. Lung cancer patients with a history of cigarette smoking can exhibit a deletion in the small arm of chromosome 3p21.3, which has several tumor suppressor genes such as LIMD [9]. In a study by Jamsai et al. the gene-trapped embryonic stem cell line was used to generate $R b m 5$ deficient $\left(^{-/}\right)$mice. When treated with the cigarette carcinogen NNK (4-[Methyl(nitroso)amino]-1-(3-pyridinyl)-1-butanone), these mice developed more aggressive cancers compared with wild-type mice with an increased number of adenocarcinoma nodules. Evidently, $R b m 5$ acts as a tumor suppressor gene in cigarette smokers [10].

Elucidating the role of the protumor microenvironment and hallmark immune signatures is essential in developing novel therapies for lung cancer. To study the role of the protumor microenvironment in lung cancer, Faget $e$ al. used Kras and p53 (KP) driven lung adenocarcinoma mice crossed with Rag1 gene to demonstrate that $\mathrm{Gr}^{+}$neutrophil deletion leads to enhanced tumor growth and reduced the efficacy of programmed cell death-1 immunotherapy [11]. They also showed that increased angiogenesis was associated with neutrophils, which promote hypoxia and Snail production that in turn causes tumor progression [11]. The use of cell-free DNA and circulating tumor DNA is gaining attention as promising biomarkers for lung cancer. This coupled with the use of Cre-regulated genetically modified mice such as Kras ${ }^{L o x-G 12 D}, K_{r a s}{ }^{L S L-G 12 D}$ and $K r a s^{W T}$ and using techniques like micro-computed tomography and droplet digital PCR assay, facilitates the detection of pre-malignancy of lung adenocarcinoma, which can be a promising diagnostic tool [12]. Conditional triple deficient $\mathrm{Rb} / \mathrm{p} 53 / \mathrm{p} 130^{\text {flox }} /$ flox mice that develop SCLC with Hes1 transcriptional factor showed that the tumors had notch-active protumorigenic (non-neuroendocrine) cells that were generated from neuroendocrine cells. Inhibition of transcriptional repressor REST in notch signalling prevented cell differentiation in SCLC. Thus, notch inhibitors along with chemotherapy may be an effective therapy for SCLC [13].

The role of lung microbiota in communication between bacteria and inflammation is gaining attention. Jin et al. showed an interesting correlation between microbiota, inflammation and lung adenocarcinoma [14]. In KP mice it was observed that microbiota promote adenocarcinoma with increased cytokine expression via promotion of $\mathrm{V} \gamma 6+\mathrm{V} \delta 1+\mathrm{T}(\gamma \delta \mathrm{T})$ cells. Thus, alteration in the pathway of microbiota mediated $\gamma \delta \mathrm{T}$ cell activation and proliferation offers it as a therapeutic target [14]. Furthermore, kinases were reported to be tumor markers, including proto-oncogene ROS1-receptor tyrosine kinase 1 - which has an important role in NSCLC progression [15]. Notably, Arai et al. developed an EZR-ROS1 transgenic mouse model to study the role of the EZR-ROS1 fusion gene [15]. The authors first fused the $E Z R$ and $R O S 1$ genes via the expression of a fusion kinase in NIH3T3 cells, and then injected these cells into nude mice, which resulted in the rapid development of early stage multiple adenocarcinomas. This new model can be used to study therapeutic agents for patients expressing ROS1 rearrangements [15].

The majority of mutations occurring in lung cancer models are Kras dependent, and corresponding mutated mice are widely used models in preclinical studies [16]. One profiling study on exomes and transcriptomes of Kras mutations in genetically modified mouse models suggested that a greater degree of heterogenicity evolved during tumorigenesis [17]. This indicates the need for further refinement of genetically modified animal models, which would aid more effective development of therapeutics.

\section{Chemically induced animal models of lung cancer}

Chemically induced animal models of lung cancer are increasing in importance compared with xenograft and genetically modified models due to their ease of application, rapid timeframes and efficiency in reproducibility. Recently, these models have gained attention, primarily due to major increases in the number of lung cancer cases related to cigarette smoking and other environmental carcinogens, such as polycyclic aromatic hydrocarbons and aromatic amines [18]. The major chemical carcinogens in cigarette smoke include NNK and polycyclic aromatic hydrocarbons [19]. These carcinogens have been extensively used to develop different models of lung cancer. For instance, NNK is used to induce adenocarcinoma [20] whereas N-nitroso-tris-chloroethylurea (NTCU) promotes the development of squamous cell carcinoma [21]. Merval et al. developed a novel animal model of NSCLC by inducing adenocarcinoma using diethylnitrosamine in the FVB/N (Friend leukemia virus B) mouse strain [22]. Interestingly, no mutations in the hot spot areas of EGFR and Kras genes were observed, which highlights the importance of this particular model in investigating the pathogenesis of EGFR and Kras negative tumors [22]. 
Lung epithelial cell lines such as BEAS-2B and human bronchial epithelial cells that lack p53 expression when exposed to NNK exhibited activation of IGF-1R phosphorylation via $\beta$-adrenergic receptor ( $\beta$-AR) [23]. Blocking $\beta$-AR with NNK antagonists in A/J mice with lung cancer suggests that blockade of $\beta$-AR may be an effective therapy for lung cancer [23]. In addition to NNK, inorganic phosphate (Pi) also plays a crucial role in signal transduction and expression of several genes like transcription factor $N r f 2$ during cell division [24]. However, the role of $\mathrm{Pi}$ in lung cancer pathogenesis is poorly understood. A study investigating the role of high phosphate diet for 1, 2 and 4 months in Kras mice suggested that the diet enhanced autophagy and epithelial to mesenchymal transition in early and later stages due to cellular metabolic adaptation, resulting in the decline of cancer. Overall, it is necessary to avoid high phosphate diet with early stages of lung cancer [24]. Another hallmark feature of lung cancer is chronic inflammation, Kim et al. proposed that cigarette smoke exposure to C57BL/6J and IL-17a ${ }^{-/-}$ in mice lungs causes increased Th17 differentiation with enhanced pro-Th17 and neutrophils, by analyzing blood and spleen, it was found that there was a systemic inflammation, eventually leading to intestinal inflammation [25].

The development of preclinical nonrodent lung cancer models may be crucial for the study of lung carcinogenesis that may more precisely mimic the human disease. Administration of NNK to ferrets $(50 \mathrm{mg} / \mathrm{kg}$ body weight) for 4 months, followed by a rest periods of 24, 26 and 32 weeks resulted in preneoplastic and neoplastic lesions [26]. This led to the development of tumors with higher numbers of macrophages and increased expression of $\alpha 7$ nicotinic acetylcholine receptors, which replicates observations in humans. Thus, non-rodent models could be used to study the progression of lung cancer in conjunction with laboratory rodents [26]. In addition to carcinogens, genetically modified A549 cells with hNIS gene expression and an imaging reporter ${ }^{99 \mathrm{~m}} \mathrm{Tc}$-pertechnetate $\left({ }^{99 \mathrm{~m}} \mathrm{TcO}^{4-}\right)$ cells have also been used to create a new orthotopic xenograft lung cancer model. Engrafting these cells to athymic nude mice results in the development of tumors, and the level of tumor progression was measured using single proton emission computed tomography/CT (SPECT/CT) with ${ }^{99} \mathrm{TcO}^{4-}$. This enabled the identification of tumor mass at different time points and highlights the importance of this orthotopic xenograft model for use in monitoring the effects of potential anticancer therapies longitudinally [27]. In order to study the cellular and molecular changes which occur during chemically induced lung adenocarcinoma, Spella et al. developed a C57BL/6 mice model of lung adenocarcinoma by exposing tobacco carcinogen urethane via intraperitoneal injection for 10 weeks. This study showed that airway epithelial cell were more sensitive to $\mathrm{Kras}^{\mathrm{Q} 61 \mathrm{R}}$ mutations compared to alveolar type II cells, suggesting that tobacco-induced lung adenocarcinoma starts in airway epithelial cells [28].

\section{Conclusion}

Collectively, the analysis of genetically modified and chemically induced animal models of lung cancer has progressed the understanding of lung cancer pathogenesis. Lung cancer in animal models progresses in a relatively short time frame and under extremely controlled conditions and aids the identification of most relevant oncogenic pathways, which could be novel drug targets. Understanding the genetic changes that occur during cancer progression is complex and depends upon the type of cancer and the stimulus. There remains a lack of robust short-term, easily reproducible, cigarette smoking-induced mouse model which replicate similar clinical features of human lung cancer.

\section{Financial \& competing interests disclosure}

Kamal Dua is supported by the Graduate School of Health, University of Technology Sydney and the Sydney Partnership for Health, Education, Research and Enterprise (SPHERE) for the TRIPLE I CAG Secondment/Exchange grant. Philip M Hansbro and Chantal Donovan are supported by a fellowship from the National Health and Medical Research Council of Australia (NHMRC \#1079187 and \#1120152). Philip M Hansbro would like to acknowledge the Cancer Council of NSW for their funding. Keshav Raj Paudel is supported by a fellowship from the Prevent Cancer Foundation (PCF)/International Association for the Study of Lung Cancer (IASLC). Vamshikrishna Malyla is supported by the Graduate School of Health and University of Technology Sydney (UTS President's scholarship and UTS International Research Scholarship). The authors have no other relevant affiliations or financial involvement with any organization or entity with a financial interest in or financial conflict with the subject matter or materials discussed in the manuscript apart from those disclosed.

No writing assistance was utilized in the production of this manuscript. 


\section{References}

1. Bray F, Ferlay J, Soerjomataram I, Siegel RL, Torre LA, Jemal A. Global cancer statistics 2018: GLOBOCAN estimates of incidence and mortality worldwide for 36 cancers in 185 countries. CA Cancer J. Clin. 68(6), 394-424 (2018).

2. Rodriguez-Canales J, Parra-Cuentas E, Wistuba II. Diagnosis and molecular classification of lung cancer. In: Lung Cancer. Reckamp K (Ed.). Springer, Berlin, Germany, 25-46 (2016).

3. Gazdar AF, Bunn PA, Minna JD. Small-cell-lung cancer: what we know, what we need to know and the path forward. Nat. Rev. Cancer 17(12), 725-737 (2017).

4. Kwon M-C, Berns A. Mouse models for lung cancer. Mol. Oncol. 7(2), 165-177 (2013).

5. Caramori G, Ruggeri P, Mumby $S$ et al. Molecular links between COPD and lung cancer: new targets for drug discovery? Expert Opin. Ther. Targets 23(6), 539-553 (2019).

6. Rajendra K, Shukla SD, Gautam SS, Hansbro PM, O'toole RF. The role of environmental exposure to non-cigarette smoke in lung disease. J. Transl. Med. 7(1), 39 (2018).

7. Kwong FNK, Nicholson AG, Harrison CL, Hansbro PM, Adcock IM, Chung KF. Is mitochondrial dysfunction a driving mechanism linking COPD to non-small-cell lung carcinoma? Eur. Respir. Rev. 26(146), 170040 (2017).

8. Wu Q, Tian Y, Zhang J et al. In vivo CRISPR screening unveils histone demethylase UTX as an important epigenetic regulator in lung tumorigenesis. PNAS 115(17), E3978-E3986 (2018).

9. Sharp TV, Al-Attar A, Foxler DE et al. The chromosome 3p21. 3-encoded gene, LIMD1, is a critical tumor suppressor involved in human lung cancer development. PNAS 105(50), 19932-19937 (2008).

10. Jamsai D, Watkins DN, O'connor AE et al. In vivo evidence that RBM5 is a tumour suppressor in the lung. Sci. Rep. 7(1), 16323 (2017).

11. Faget J, Groeneveld S, Boivin G et al. Neutrophils and snail orchestrate the establishment of a pro-tumor microenvironment in lung cancer. Cell Rep. 21(11), 3190-3204 (2017).

12. Rakhit CP, Trigg RM, Le Quesne J et al. Early detection of pre-malignant lesions in a KRASG12D-driven mouse lung cancer model by monitoring circulating free DNA. Dis. Model Mech. 12(2), dmm036863 (2019).

13. Lim JS, Ibaseta A, Fischer MM et al. Intratumoural heterogeneity generated by Notch signalling promotes small-cell-lung cancer. Nature 545(7654), 360-364 (2017).

14. Jin C, Lagoudas GK, Zhao C et al. Commensal microbiota promote lung cancer development via $\gamma \delta \mathrm{T}$ cells. Cell 176(5), 998-1013 (2019).

15. Arai Y, Totoki $\mathrm{Y}$, Takahashi $\mathrm{H}$ et al. Mouse model for ROS1-rearranged lung cancer. PLoS ONE 8(2), e56010 (2013).

16. Prior IA, Lewis PD, Mattos C. A comprehensive survey of Ras mutations in cancer. Cancer Res. 72(10), 2457-2467 (2012).

17. Chung W-J, Daemen A, Cheng JH et al. Kras mutant genetically engineered mouse models of human cancers are genomically heterogeneous. PNAS 114(51), E10947-E10955 (2017).

18. Vineis P, Pirastu R. Aromatic amines and cancer. Cancer Causes Control 8(3), 346-355 (1997).

19. Liu Y, Yin T, Feng Y et al. Mammalian models of chemically induced primary malignancies exploitable for imaging-based preclinical theragnostic research. Quant. Imag. Med. Surg. 5(5), 708-729 (2015).

20. Ge G-Z, Xu T-R, Chen C. Tobacco carcinogen NNK-induced lung cancer animal models and associated carcinogenic mechanisms. Acta. Biochim. Biophys. Sin. 47(7), 477-487 (2015).

21. Wang Y, Zhang Z, Yan Y et al. A chemically induced model for squamous cell carcinoma of the lung in mice: histopathology and strain susceptibility. Cancer Res. 64(5), 1647-1654 (2004).

22. Mervai Z, Egedi K, Kovalszky I, Baghy K. Diethylnitrosamine induces lung adenocarcinoma in FVB/N mouse. BMC Cancer 18(1), 157 (2018).

23. Min H-Y, Boo H-J, Lee HJ et al. Smoking-associated lung cancer prevention by blockade of the beta-adrenergic receptor-mediated insulin-like growth factor receptor activation. Oncotarget 7(43), 70936-70947 (2016).

24. Beck Jr GR, Moran E, Knecht N. Inorganic phosphate regulates multiple genes during osteoblast differentiation, including Nrf2. Exp. Cell Res. 288(2), 288-300 (2003).

25. Diehl G, Kim M, Gu B et al. Cigarette smoke induces intestinal inflammation via a Th17 cell-neutrophil axis. Front. Immunol. 10, 75 (2019).

26. Aizawa K, Liu C, Veeramachaneni S, Hu K-Q, Smith DE, Wang X-D. Development of ferret as a human lung cancer model by injecting 4-( $N$-methyl- $N$-nitrosamino)-1-(3-pyridyl)-1-butanone (NNK). Lung Cancer 82(3), 390-396 (2013).

27. Price DN, Mcbride AA, Anton $\mathrm{M}$ et al. Longitudinal assessment of lung cancer progression in mice using the sodium iodide symporter reporter gene and SPECT/CT imaging. PloS ONE 11(12), e0169107 (2016).

28. Spella M, Lilis I, Pepe MA et al. Club cells form lung adenocarcinomas and maintain the alveoli of adult mice. eLife 8, e45571 (2019). 